\title{
e-Phaïstos
}

e-Phaïstos Revue d'histoire des techniques / Journal of the history of technology

VIII-1 | 2020

Écomusée : une expansion internationale

\section{Les bois sacrés et les sites associés de la commune de Koudougou au Burkina Faso : des atouts pour la création d'un écomusée}

Sacred Woods and Associated Sites in the Commune of Koudougou in Burkina

Faso: Assets for the Creation of an Eco-museum

\section{Noaga Birba}

\section{OpenEdition}

Journals

Édition électronique

URL : http://journals.openedition.org/ephaistos/7667

DOI : 10.4000/ephaistos.7667

ISSN : 2552-0741

Éditeur

IHMC - Institut d'histoire moderne et contemporaine (UMR 8066)

Référence électronique

Noaga Birba, "Les bois sacrés et les sites associés de la commune de Koudougou au Burkina Faso des atouts pour la création d'un écomusée », e-Phaïstos [En ligne], VIII-1 | 2020, mis en ligne le 29 avril 2020, consulté le 05 mars 2021. URL : http://journals.openedition.org/ephaistos/7667 ; DOI : https:// doi.org/10.4000/ephaistos.7667

Ce document a été généré automatiquement le 5 mars 2021

Tous droits réservés 


\title{
Les bois sacrés et les sites associés de la commune de Koudougou au Burkina Faso : des atouts pour la création d'un écomusée
}

\author{
Sacred Woods and Associated Sites in the Commune of Koudougou in Burkina \\ Faso: Assets for the Creation of an Eco-museum
}

Noaga Birba

1 Située dans la région du centre-ouest du Burkina Faso, la commune de Koudougou regorge d'innombrables sites culturels et naturels, témoins tangibles de son identité historique et culturelle. Parmi les biens culturels qu'elle abrite, les bosquets sacrés, qui sont une association du culturel et du naturel, demeurent les plus importants. Chaque secteur de la commune possède ses bosquets-sacrés. Ils doivent leur survie au fait d'être associés à la vision spirituelle des populations et à toutes les pratiques y afférant ainsi qu'au fait d'être frappés d'interdits perçus comme des codes implicitement édictés avec la complicité de la «vision métaphysique du monde et au compte des systèmes traditionnels de protection de l'environnement. Ainsi, en même temps qu'ils constituent un poumon vert pour la ville de Koudougou qui les abrite, ils se présentent du point de vue historique et patrimonial comme un lieu de mémoire susceptible de livrer des informations sur l'histoire culturelle et sociale des communautés locales, un patrimoine culturel physique qu'il convient de conserver et de valoriser » (SFG1988 $2015: 56$ et 92 ).

2 En Afrique noire, les religions traditionnelles accordent une grande importance aux lieux sacrés et de mémoire. Ces religions se caractérisent par un ensemble de croyances locales, fortement ancrées dans les mythes fondateurs des communautés. Elles accordent une place importante aux esprits, aux ancêtres et à certains êtres vivants. Les bois sacrés qui constituent un instrument de la spiritualité traditionnelle sont le lieu de cosmogonie suprême, le trait d'union entre l'ici et l'ailleurs spirituel, le lieu de transition entre le monde des humains et celui des non humains. Le caractère sacré des 
bois concerne sa dimension historique (Elamé 2003). Ils sont considérés comme les premiers lieux de résidence et / ou d'inhumation des communautés autochtones. La multitude des bosquets sacrés, une des composantes du patrimoine de la commune, en est la résultante. En effet, le couvert végétal des bosquets sacrés dans la ville de Koudougou est dominé par des espèces telles que l'Acacia pennata, l'Anogneissus leicarpus, le Pilostygma thonningii, le Comretum micranthum, l'Adansonia digitata etc. en dessous desquels se développe une variété d'herbacées avec du bois morts dont le prélèvement est interdit. Cela a permis l'épanouissement de la strate ligneuse.

Certains bosquets sacrés partagent leurs territoires avec des collines, elles-mêmes sacrées. Les différents sites sont dans leur ensemble très menacés. Il s'avère alors impérieux de mener des actions de conservation et de valorisation de ce patrimoine tant culturel que paysager, archéologique et historique dans une perspective de développement durable. En effet, ce patrimoine peut bien être préservé à travers la création d'un écomusée communal avec comme objectif principal de le valoriser dans son intégrité. Cet écomusée sera un cadre idéal pour exposer le territoire communal de Koudougou, son environnement culturel et naturel ainsi que les métiers traditionnels qui s'y déroulent. En effet, un écomusée est un instrument qu'un pouvoir et une population conçoivent, fabriquent et exploitent ensemble. Ce pouvoir, avec les experts, les facilités, les ressources qu'il fournit. Cette population, selon ses aspirations, ses savoirs, ses facultés d'approche (Rivière 1989 :108). Cette définition de l'écomusée met au centre l'approche participative et l'implication réelle de tous les acteurs. Elle préconise que l'écomusée soit conçu et exploité par le pouvoir (autorités décentralisées et déconcentrées) et la population (les communautés locales).

Dans cette étude, nous verrons comment s'opère le processus de protection et de valorisation face à la multitude de défis qui menace ce patrimoine.

5 L'objectif de cet article est donc de mener une réflexion sur les facteurs principaux de dégradation des sites de même que la possibilité de création d'un écomusée dans la ville de Koudougou aux fins de préserver les biens culturels et naturels, actuellement en proie aux besoins multiples des sociétés modernes. Pour ce faire, nous avons adopté une méthodologie d'approche pour mieux appréhender notre sujet de recherche. Cette méthodologie s'est basée prioritairement sur les enquêtes ethnographiques menées auprès des populations locales de la ville de Koudougou et des prospections archéologiques. La démarche a été complétée par une exploitation des sources écrites susceptibles de livrer des informations relatives au sujet traité.

6 Cet article s'organise autour de trois points essentiels. Le premier grand point de notre réflexion s'intéresse aux différents sites patrimoniaux de la ville de Koudougou. Le deuxième point présente les menaces auxquelles sont confrontés le patrimoine culturel et naturel de Koudougou et le troisième, enfin, analyse les actions concrètes qui doivent être menées pour sauvegarder et valoriser les différents sites dans une perspective de développement durable.

\section{Le cadre d'étude}

7 La ville de Koudougou est la capitale de la région du Centre-Ouest et chef-lieu de la province du Boulkiemdé. Elle est située à $97 \mathrm{~km}$ à l'ouest de Ouagadougou, dans le Plateau Central, à $282 \mathrm{~km}$ de Bobo et $130 \mathrm{~km}$ de Dédougou et est reliée à la capitale, Ouagadougou, par la route nationale bitumée ${ }^{\circ} 14$ de $40 \mathrm{~km}$ et la Route Nationale ${ }^{\circ} 1$ 
sur $60 \mathrm{~km}$. Elle est aussi en communication avec Ouagadougou et Bobo par le chemin de fer qui relie Ouagadougou à Abidjan. Le site de la ville correspond aux coordonnées géographiques $2^{\circ} 37^{\prime}$ de longitude ouest et $12^{\circ} 25^{\prime}$ de latitude nord. L'agglomération urbaine s'étale sur plus de $7 \mathrm{~km}$ d'Est en Ouest, de part et d'autre de la voie ferrée. Avec la communalisation intégrale de 2006, le territoire communal compte en plus des dix secteurs de la ville, quinze villages rattachés, poussant ainsi les limites communales qui s'étendent désormais sur une superficie de $580 \mathrm{~km}^{2}$ au lieu de $272 \mathrm{~km}^{2}$ auparavant.

Sur le plan physique, la géologie s'intègre à celle de la région du centre-ouest. Elle est formée en majorité de roches précambriennes (granites, de complexes schisteux birrimiens, de formations sédimentaires et de roches vertes (Kienon-Kaboré 2003 :41). La zone est soumise à un climat continental sec soudano-sahélien caractérisé par deux saisons aux durées inégales. Il s'agit de la saison sèche qui va de septembre à mai et de la saison des pluies qui s'étale de mai à septembre. Ce climat a donné une teinte particulière à la végétation qui va de la brousse tigrée à la savane arborée. Le morcellement actuel de la végétation laisse apparaitre quelques forêts éparses. Témoins d'un couvert végétal autrefois luxuriant, sa survivance est intimement liée à une forme de gestion traditionnelle de l'environnement dans laquelle les pratiques cultuelles sont l'épine dorsale. Les populations y associent leur vie religieuse, leur vision du monde métaphysique et parfois même leur histoire.

Du point de vue historique, la commune de Koudougou est investie depuis des siècles par diverses populations ; elle a été, comme l'ensemble de la région, l'objet de plusieurs convoitises et une zone de convergence de différents groupes ethniques. Ces groupes sociaux ont laissé des traces matérielles qui témoignent de l'occupation humaine ancienne de la commune de Koudougou depuis la préhistoire jusqu'aux périodes historiques.

Fig.1 : Localisation de la commune de Koudougou au Burkina Faso

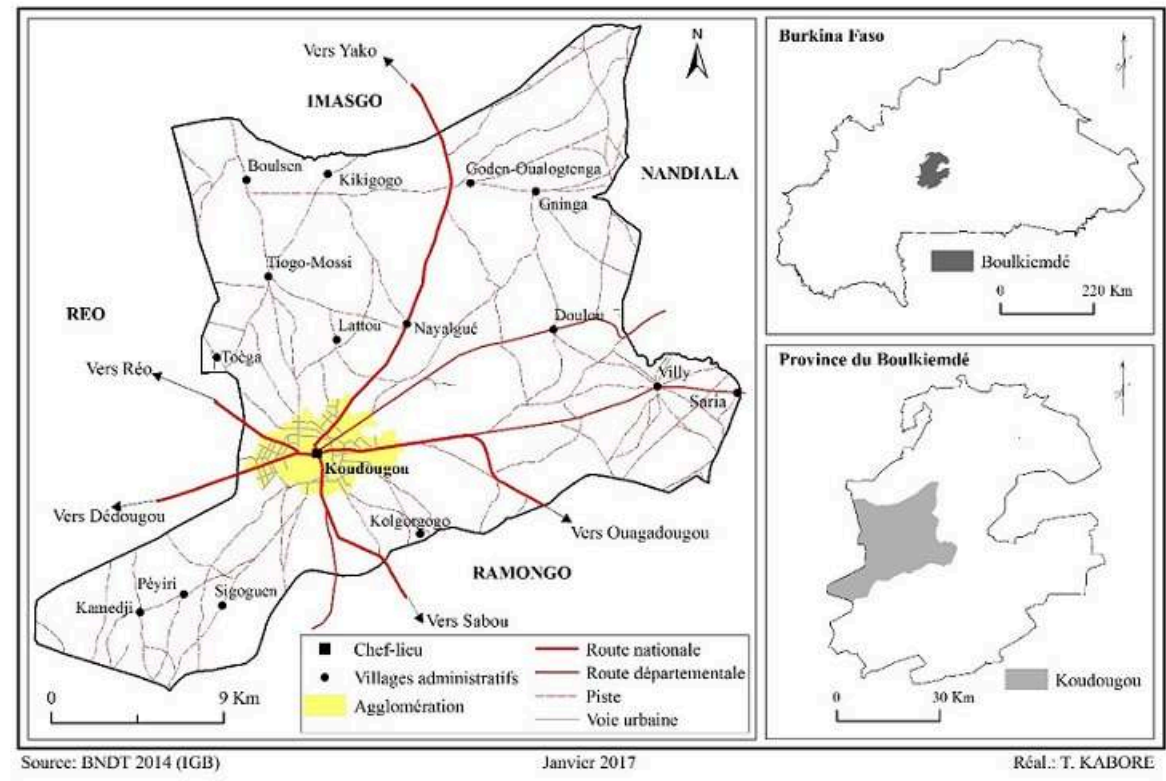




\section{Les composantes des sites du patrimoine culturel de la commune de Koudougou}

10 Les différents biens culturels identifiés comprennent les bois sacrés, les sites archéologiques et les monuments historiques. Ils sont tous localisés dans les différents secteurs de la ville de Koudougou.

Fig. 2 : Répartition spatiale des sites patrimoniaux de la ville de Koudougou

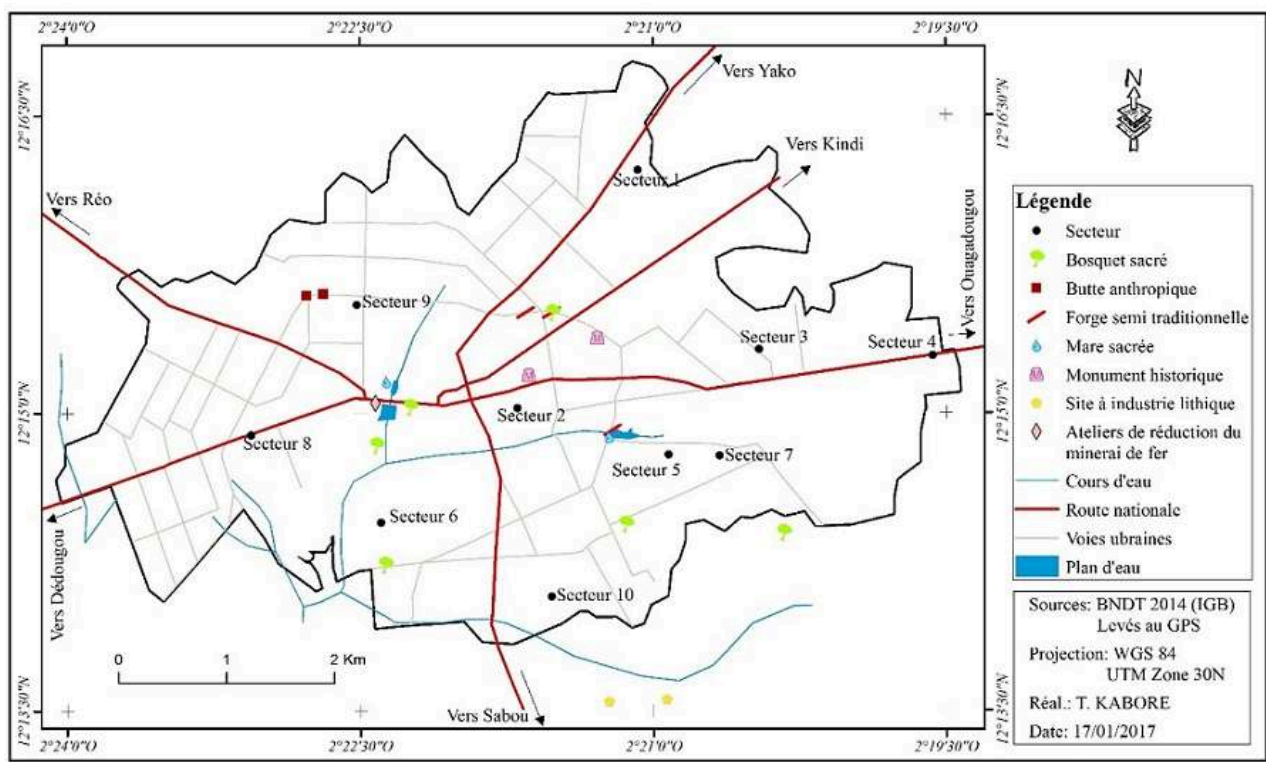

Source : BNDT 2014 (IGB). Réal. T. Kabore, Janvier 2017

\section{Les bois sacrés de la ville de Koudougou : charge historique et dimension patrimoniale}

11 Parler du patrimoine culturel de l'Afrique sans évoquer les paysages culturels, c'est omettre un pan conséquent de ce patrimoine, l'amputer d'une composante très importante et qui passe souvent pour être la plus représentative. Cela est lié au fait qu'elle est particulièrement riche en paysages culturels vivants (Barillet, Joffroy et Longuet $2006: 15$ ), résultat d'une compréhension harmonieuse de la relation entre l'homme et la nature. La conséquence en a été la formation de lieux respectés et qui sont objets de culte. Dans la plupart des contrées du Burkina Faso, cette réalité, vécue depuis des siècles, anime le quotidien des populations. Les éléments naturels tels les collines, les mares, les rochers, les bosquets, etc. sont parfois sacralisés, garantissant aussi bien la survie d'un patrimoine immatériel vivant que la protection d'un environnement en proie aux besoins multiples des sociétés modernes.

Situé dans la zone sahélienne, le Burkina Faso est reconnu comme la zone de savane par excellence. Si par endroit la végétation doit sa survie aux politiques nationales relatives 
à la protection de l'environnement, surtout avec les Engagements Nationaux dont une des options était de réhabiliter le couvert végétal ${ }^{1}$, il y a lieu de signaler qu'en marge de tout cela la vision traditionnelle de l'environnement a été et est toujours d'un grand apport. En effet, elles sont rares les localités du Burkina Faso à ne pas posséder de forêts ou de bosquets sacrés, indépendamment des politiques nationales et auxquelles elles sont sans équivoque antérieures. Ces isolats de forêts, tout en garantissant une psychologie vitale aux populations parce qu'abritant les esprits protecteurs du lieu et incarnant des valeurs culturelles et parfois économiques ${ }^{2}$, sont le creuset d'une biodiversité pouvant être exploitée par les collectivités locales.

Dans notre zone d'étude, la ville de Koudougou, le rapport étroit entre l'homme et la nature a abouti à la formation de paysages culturels dont les bosquets sacrés. En moyenne, chacun des six quartiers (Bourkina, Issouka, Dapoya, Godin, Issouka et Sogpelcé) qui constituent la ville en possède au moins deux.

Le tableau ci-dessous permet d'avoir une idée de la diversité des essences végétales que l'on trouve dans les bosquets sacrés. Ces essences sont constituées d'arbres, d'arbustes et d'herbacées. L'ensemble de ces espèces forme de grands buissons appelés kango en langue locale mooré. Ces espaces végétaux forment des lieux de culte et de mémoires pour les différents quartiers de la ville de Koudougou. Ils constituent des matériaux importants pour la reconstitution de l'histoire des populations actuelles.

Tableau 1 : Récapitulatif des espèces végétales rencontrées dans les bosquets sacrés.

\begin{tabular}{|l|l|l|}
\hline Noms Scientifiques & Noms locaux en mooré & Familles \\
\hline Azadirachta indica & & Meliaceae \\
\hline Diospyros mespiliformis & Gaanga & Ebenaceae \\
\hline Tectona grandis & & Verbenaceae \\
\hline Gardenia erubescens & Souba & Rubiaceae \\
\hline Adansonia digitata & Toèga & Bombacaceae \\
\hline Acacia nilotica & Pèg-nenga & Mimosaceae \\
\hline Khaya senegalensis & Kouka & Meliaceae \\
\hline Faidherbia albida & Zaanga & Mimosaceae \\
\hline Ficus ingens & Kuiguinga & Moraceae \\
\hline Combretum glutinosum & Koutinpagdré & Combretaceae \\
\hline Anogeissus leiocarpa & Siiga & Combretaceae \\
\hline Acacia penneta & Kango & Mimosaceae \\
\hline Guiera senegalensis & Wilinwiga & Combretaceae \\
\hline Cassia sieberiana & Koumbri-saka & Mimosaceae \\
\hline
\end{tabular}

Source : Enquête de terrain, juin 2017 
Fig.3. Bois sacré du quartier Issouka

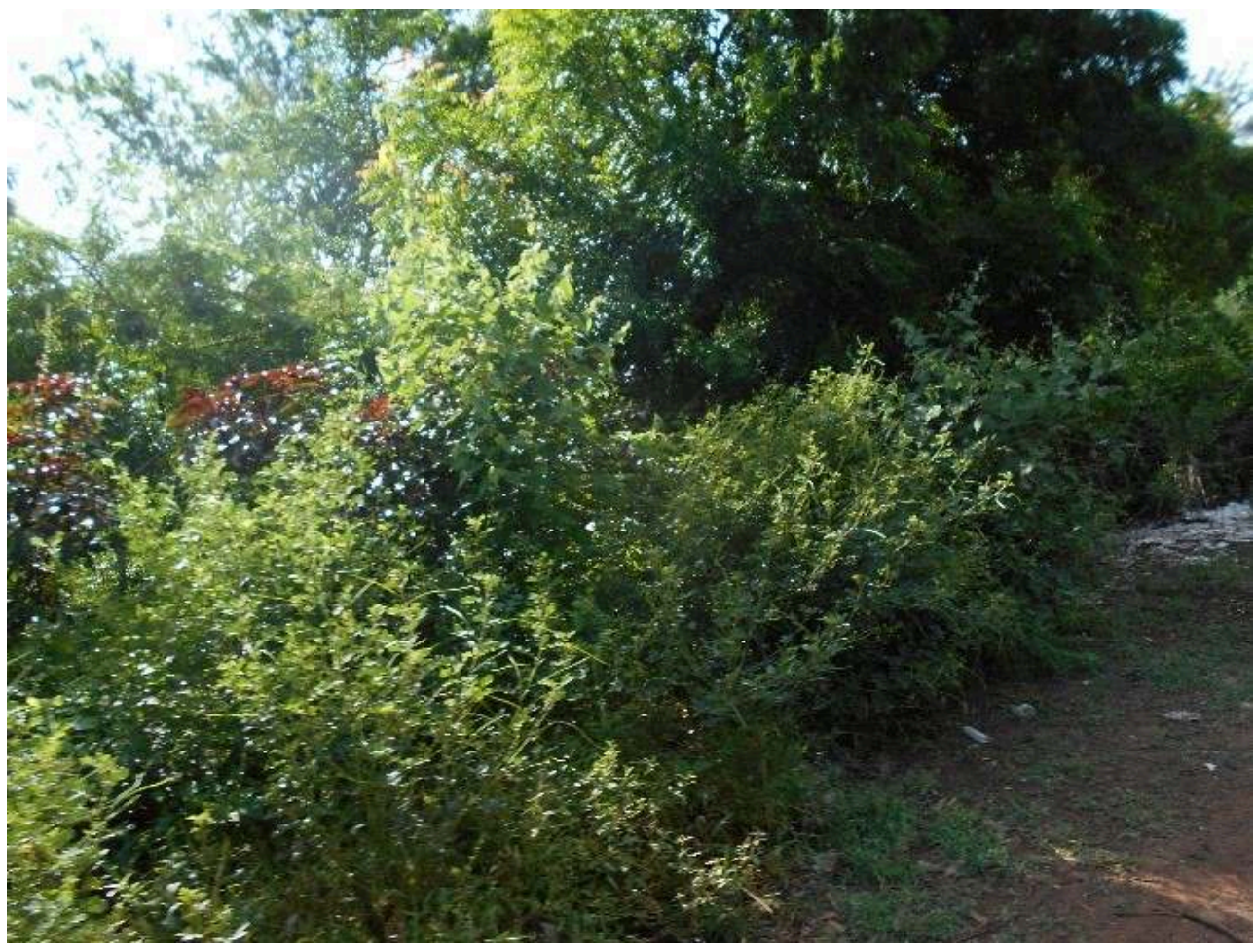

Photo : Birba N., juillet 2016

Fig.4. Bois sacré du quartier Sogpelcé

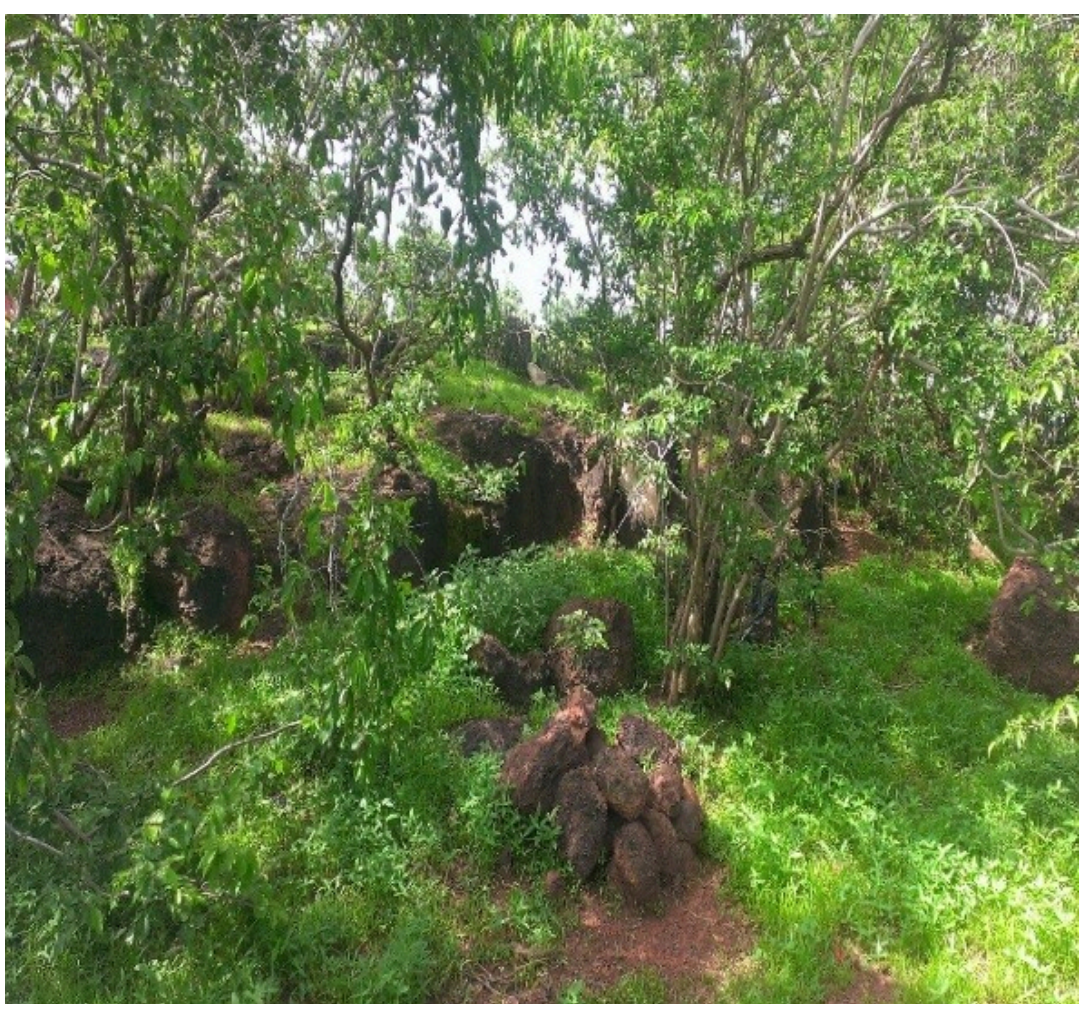

Photo : Birba N., juillet 2016 


\section{La charge historique des bois sacrés} sites d'habitats de certaines familles autochtones. Selon Yaméogo Tenga ${ }^{3}$, le géant baobab (Adansonia digitata), localisé dans le quartier Bourkina est un arbre mémoire et vénéré par tout le quartier. Il matérialiserait le premier emplacement des habitats des Yaméogo du quartier Bourkina. Cet arbre sert de repère historique; il est devenu l'emblème de la mairie de Koudougou. De même, les deux bosquets sacrés, à savoir les sites nommés Nabite Naab Tanga et Dekonwonlgzanga, identifiés dans le quartier Sogpelcé seraient, aux dires du vieux Zongo Tiga ${ }^{4}$, l'emplacement des premières résidences des Yaméogo de Sogpelcé. Les arbres géants qui s'y trouvent sont considérés comme arbres mémoires et sacrés. Dans les quartiers de la ville de Koudougou, des exemples similaires foisonnent. La plupart des bois sacrés identifiés appartiennent à des familles ou groupes de familles et sont chargés d'histoire. Ils représentent un héritage des générations fondatrices des communautés autochtones de Koudougou et ont une valeur qui les rend digne de protection et de valorisation. La culture traditionnelle des communautés tire en grande partie sa vitalité, sa richesse spirituelle, de la relation qu'elle entretient avec ces sanctuaires boisés. Ils demeurent des espaces de médiation, de culte et de recueillement pour les populations autochtones.

Ces bosquets sacrés sont des réceptacles et des canaux de communication et de communion entre les vivants et leurs dieux, leurs ancêtres. Chaque année, des rites y sont effectués par les communautés. Ce sont des lieux de mémoire qui permettent de sauvegarder les liens historiques des différentes populations qui en sont les propriétaires.

17 Au-delà de leur importance historique, les bois sacrés constituent un patrimoine culturel et naturel de grande importance pour la commune de Koudougou. 
Fig.5. Arbre sacrificiel du quartier Bourkina (Andasonia digitata-baobab)

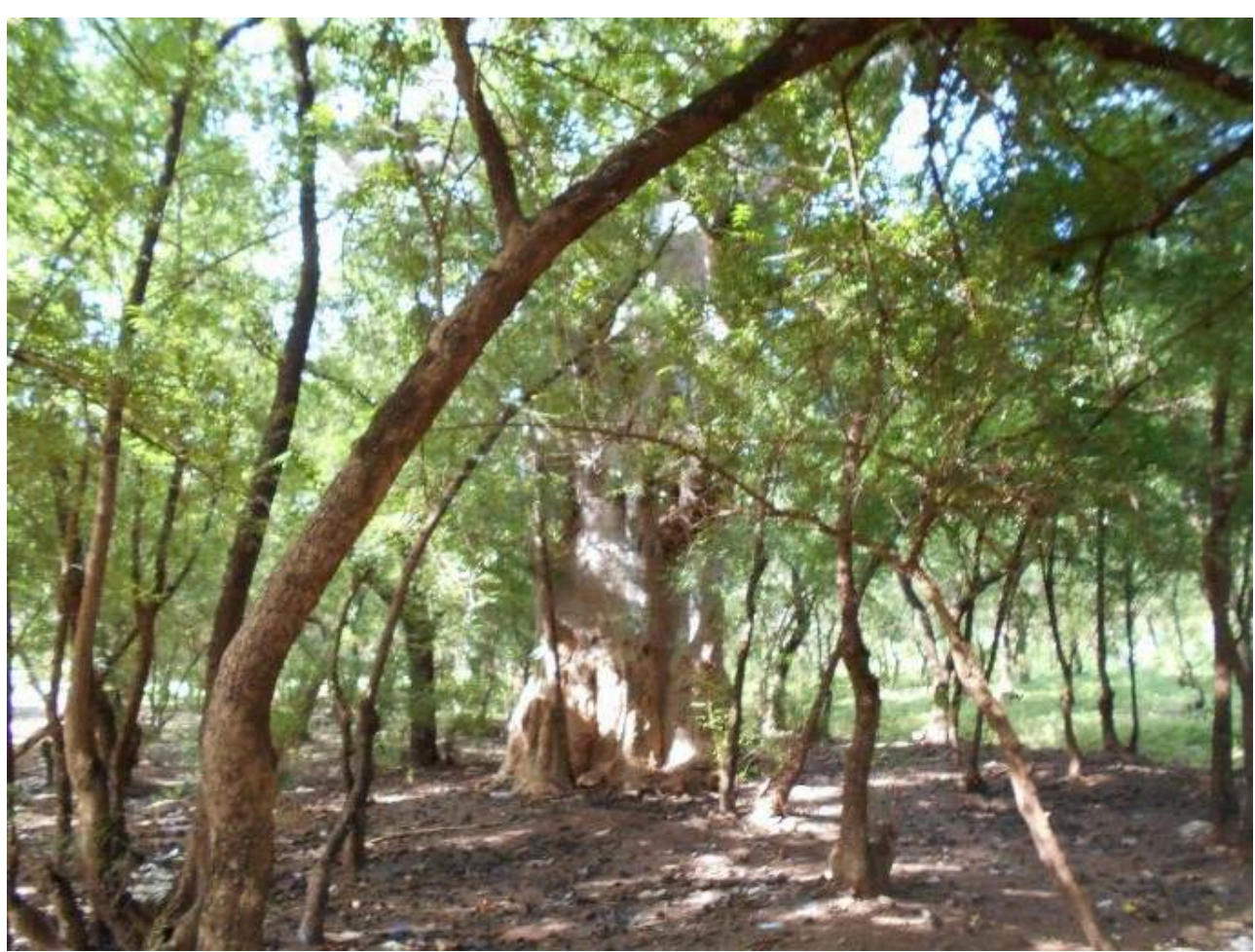

Photo : Birba N., septembre 2015

Fig.6. Arbre sacré du quartier Sogpelcé

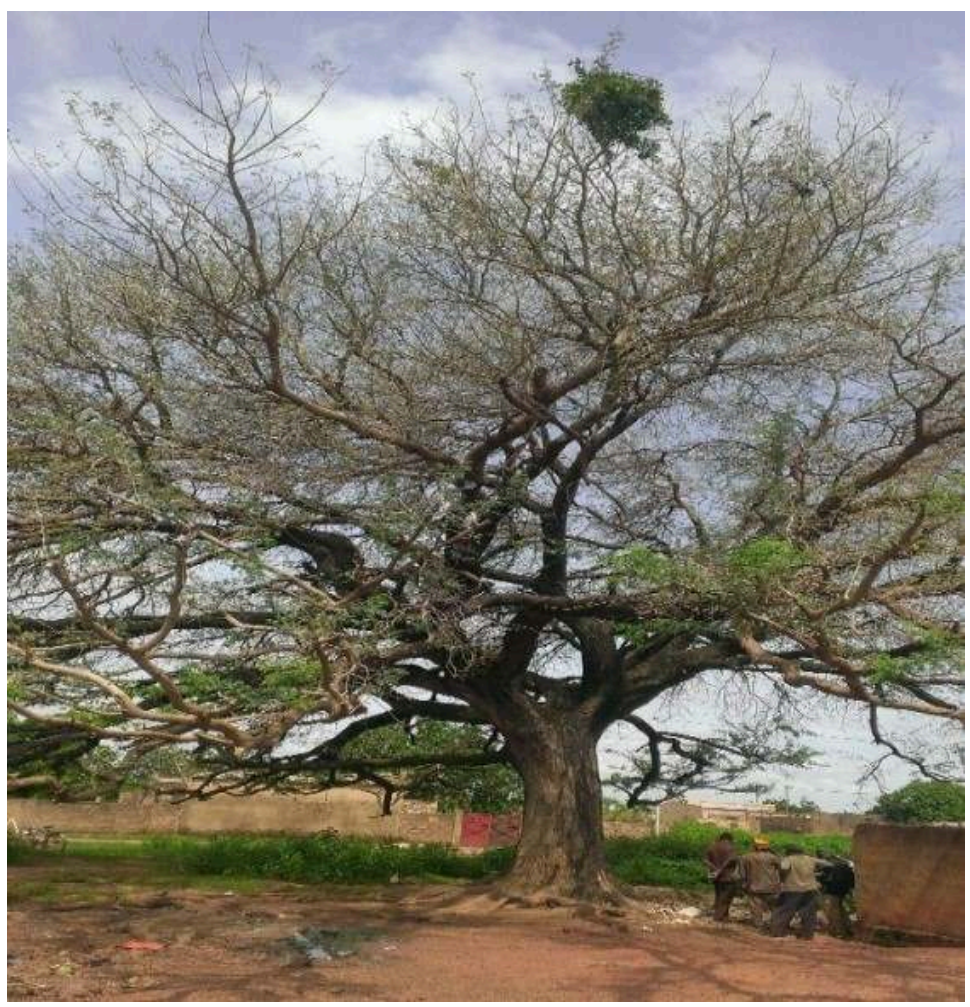

Photo : Birba N., septembre 2015 


\section{La dimension patrimoniale des bois sacrés}

18 Selon la définition du Conseil de l'Europe, le patrimoine est «un ensemble de ressources héritées du passé que des personnes considèrent, par-delà le régime de propriétés des biens, comme un reflet et une expression de leurs valeurs, croyances, savoirs et traditions en continuelle évolution. Cela inclut tous les aspects de l'environnement résultant de l'interaction dans le temps entre les personnes et les lieux ${ }^{5} »$. Ainsi, tout patrimoine est un héritage qui conserve des valeurs qui doivent être transmises aux générations futures. En cela, les bois sacrés de Koudougou constituent à la fois un patrimoine culturel et naturel.

n effet, le Comité intergouvernemental pour la protection du patrimoine mondial culturel et naturel associe depuis 1992 les éléments naturels et culturels et aboutit à la définition de "paysage culturel " (Adjanohoun 1998). Trois catégories de paysages culturels sont reconnues par le Comité du patrimoine mondial (16 ession,1992): les paysages créés (jardins, parcs), les paysages évolutifs (reliques ou vivants, ils sont associés aux modes de vie traditionnels) et les paysages associatifs (association de phénomènes religieux, culturels à l'élément naturel), auxquels se rattachent directement les bois sacrés (Juhé-Beaulaton 2006 :34).

20 Ainsi, les paysages culturels constituent une trame très importante dans le paysage patrimonial culturel du Burkina Faso. CEuvres conjuguées de l'homme et de la nature, ils illustrent plus particulièrement les spécificités des rapports entre les sociétés humaines et leur environnement (Barillet et al. 2006 :15). Celui-ci, dans la plupart des sociétés traditionnelles africaines, constitue une donnée plurifonctionnelle au point que dans l'esprit des populations, son caractère précieux et sa sacralité passent avant toute chose. Dans nombre de contrées moaga, la nature est associée à des images mentales représentatives de la spiritualité, de pratiques culturelles ou de traditions populaires. Là, le tangible (collines, mares, arbre isolé ou bosquets, etc.) et l'immatériel (cultes et rituels y relatives) s'imbriquent et se complètent. Ces éléments sont parfois associés à des mythes fondateurs (Le Berre 2000 :53) ou à des questions religieuses. La nature transcende ici l'aspect physique. On arrive donc à comprendre pourquoi ils bénéficient d'un traitement particulier au sein des communautés.

21 Dans la commune de Koudougou, les bois sacrés sont vus par les communautés autochtones comme une partie intrinsèque de leur identité culturelle. Ils constituent des références identitaires et sont les témoins tangibles de leur histoire. Ils portent tous les signes qui accompagnent le changement. Ce sont leurs monuments patrimoniaux et leurs musées. Enfin, ce sont leurs patrimoines culturels et naturels qu'ils doivent préserver et transmettre aux générations futures dans une perspective de développement durable.

Outre les bois sacrés, la ville de Koudougou possède d'autres éléments de paysage culturel, notamment les sites hydriques, c'est-à-dire les cours d'eau. L'on peut citer le Bul-kiongo (puits intarissable), le marigot de Bourkina et le marigot sacré « Rita-samba ». Selon les traditions orales, ces points d'eau abriteraient des divinités qui protègent la ville de Koudougou. Ils sont tous sacrés et seraient des lieux de culte. Parmi ces marigots sacrés, la mare "rita-samda » en langue locale mooré est la plus connue. Elle serait la propriété culturelle et cultuelle des Yaméogo de Sogpelcé. Elle aurait la particularité de noyer toute personne ayant commis une aberration ou qui est animée 
d'un esprit maléfique (sorcier, tueur, etc.), qui y entrerait ou s'en approcherait de trop près (Yaméogo 2015 :79).

La mare «rita-samba » est considérée comme une divinité par les populations locales. Des sacrifices solennels collectifs et des sacrifices individuels y sont effectués. Les sacrifices sont officiés par les sages du quartier Sogpelcé. La mare demeure un haut lieu de culte et d'histoire pour toute la commune de Koudougou en générale et les autochtones de Sogpelcé en particulier. C'est un paysage culturel renfermant des patrimoines qui peuvent être exploités à des fins touristiques et historiques.

Fig.7. La mare sacré « rita-samba » dans le quartier Sogpelcé

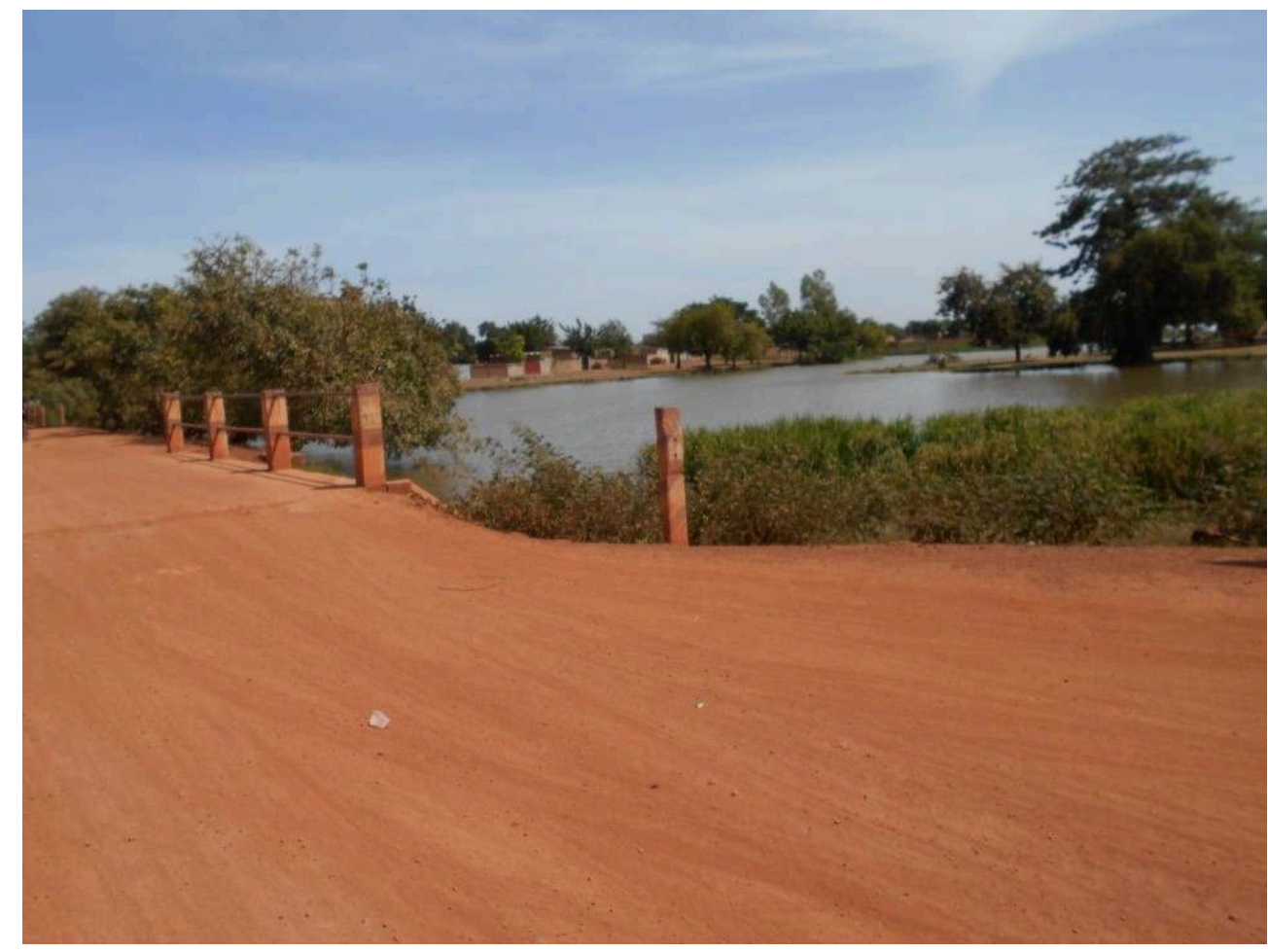

Photo : Birba N., octobre 2015

\section{Les sites archéologiques}

24 Les investigations archéologiques réalisées dans la ville de Koudougou ont permis de mettre au jour trois catégories de sites archéologiques. Ils sont caractéristiques de la préhistoire, des buttes anthropiques et des ateliers relatifs à la production ancienne du fer.

\section{Les sites préhistoriques}

Les sites préhistoriques sont caractérisés par la présence d'atelier de taille de la pierre. Ainsi, l'existence ancienne de l'homme dans la ville de Koudougou est attestée par les témoins de l'industrie lithique. Selon Koté Lassina, l'industrie lithique concerne le système technologique de l'homme, fondé sur la production d'outils en pierre, avant la découverte des métaux et alliages comme le cuivre, le bronze ou le fer (Koté 2012 :20). 
plusieurs secteurs de la ville de Koudougou, de nombreux sites à industrie lithique ont été découverts. Ces différents sites sont composés de nombreux outils lithiques, notamment des microlithes, des nucleus, des haches polies et des percuteurs taillés sur silex et sur quartz. Les travaux de Stéphane Sanou permettent aujourd'hui de situer l'occupation humaine dans la ville de Koudougou, notamment dans le quartier Sogpelcé, au paléolithique supérieur au moins (Sanou 2017).

Fig.8. Site à industrie lithique dans le quartier Sogpelcé

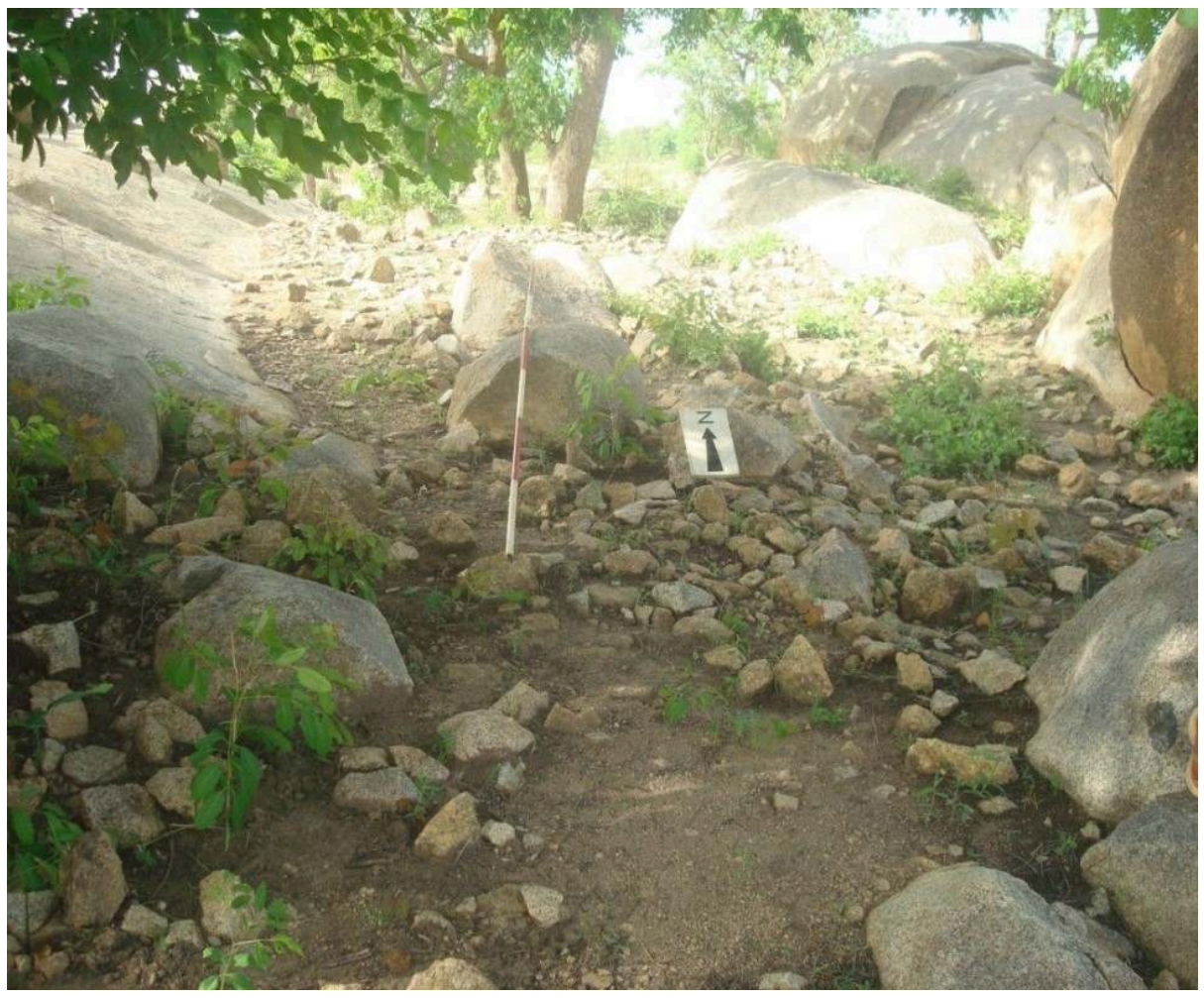

Photo : Sanou S.S. octobre 2014

\section{Les buttes anthropiques}

La présence dans un endroit donné d'une butte anthropique est un indice important de l'existence passée et/ou présente de l'homme. «Les buttes anthropiques sont des accumulations de matériaux dues à l'activité humaine. Elles sont particulièrement précieuses pour la connaissance de l'histoire et la culture des communautés humaines. Leur étude permet d'apporter des informations et précisions précieuses sur les civilisations passées. Elles furent des anciens habitats autrefois occupés par les populations actuelles de la zone ou par des peuples qui ont migré vers d'autres régions. Ces buttes anthropiques se caractérisent par la présence de nombreux tessons de céramique aux dimensions et aux formes variées, avec des décors multiples, bien conservés et parfois des outils lithiques (meules, broyeurs, des percuteurs...)» (SFG1988 2015 :49). Dans les dix secteurs de la ville de Koudougou, de nombreuses buttes anthropiques ont été mises au jour. 
Fig.9. Butte anthropique jonchée de tessons de céramique dans le quartier Bourkina

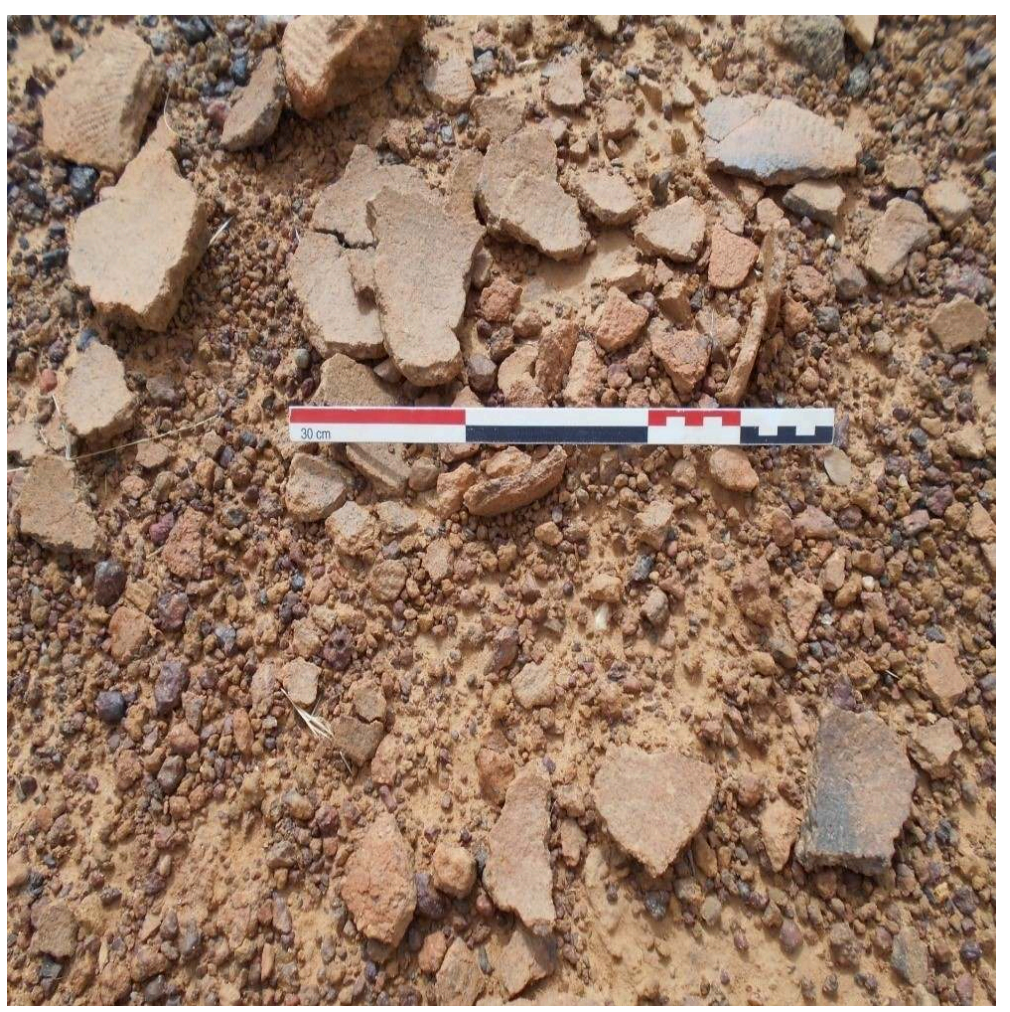

Photo : Birba N., octobre 2015

\section{Les sites de la production ancienne du fer}

En l'état actuel des recherches, le développement de la métallurgie ancienne du fer au Burkina Faso est daté autour du VIII siècle avant notre ère (Koté 2004). Dans la ville de Koudougou, cette industrie ancienne a laissé de nombreuses traces. Ces témoins métallurgiques sont constitués essentiellement d'ateliers de réduction du minerai de fer. En effet, un atelier de réduction est un espace où des fourneaux, des bases de fourneaux, des tuyères, ou des déchets du travail métallurgique (scories de fer) attestent d'une production ancienne de fer. Les sites métallurgiques identifiés dans la ville de Koudougou sont dans un état de dégradation très avancé. Les seuls vestiges encore visibles sont les bases de fourneau arasées, les fragments de parois et des épandages de scories.

Dans la ville de Koudougou, en plus des sites relatifs à la sidérurgie extractive, il existe des forges traditionnelles et semi-modernes qui sont toujours actives. Ces lieux d'apprentissage technique et cultuel constituent des biens culturels susceptibles d'être mis en valeur d'un point de vue touristique, dans le cadre d'un projet d'écomusée. 
Fig.10. Vue d'un atelier de réduction du minerai de fer dans le quartier Sogpelcé

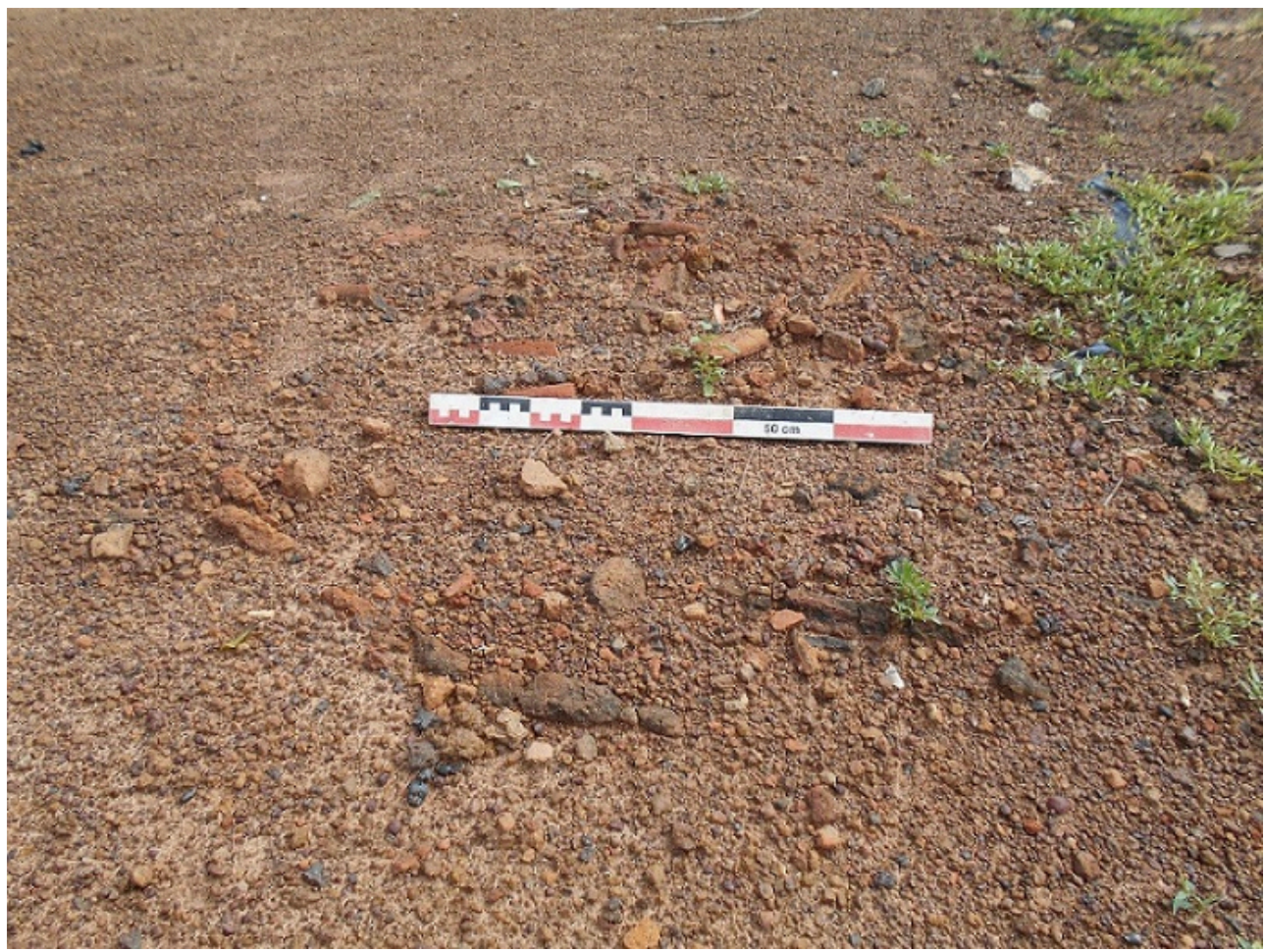

Photo : Birba N., octobre 2015

\section{Les monuments historiques de la ville de Koudougou}

Outre les bosquets sacrés et les sites archéologiques dont regorge la ville de Koudougou, l'on y note la présence de monuments historiques qui constituent son patrimoine culturel bâti. Deux principaux monuments se révèlent être les plus importants au regard de leur histoire et de leur architecture. Il s'agit de l'ancien Palais royal de Lallé et du Palais de Maurice Yaméogo. En effet, l'ancien Palais royal de Lallé est situé au sein du secteur $n^{\circ} 2$ de la commune de Koudougou, dans le quartier Zakin. L'espace qu'occupe le palais couvre environ deux hectares.

31 D'un point de vue historique, la naissance du Lallé s'est inscrite dans le cadre de la conquête et de la structuration véritable du pays moaaga amorcées par Naaba Koumdoumié ${ }^{6}$. Son fils, Naaba Kuuda ${ }^{7}$, en fut le continuateur, en multipliant les chefferies issues de sa parenté (Tiendrebeogo 1963 : 35). C'est ainsi que Lallé vit le jour grâce à l'un de ses fils, Bèega, entre la fin du XVI ${ }^{\mathrm{e}}$ siècle et le début du XVII ${ }^{\mathrm{e}}$ siècle.

A l'ouest du royaume de Ouagadougou, entre les Rimbi de Konkistenga au nord et de Kayao au sud, existaient les villages de Lay, Sao, Sourgou, Malviré, Youtinga, Boulmtinga et Nandiala (Kohler 1966). Un climat d'insécurité totale régnait dans la région, malgré le contrôle exercé concomitamment par les nanamse de Laye et de Nandiala. "En effet, les razzias étaient une pratique courante. Les nanamsés de Sourgou et de Saoa venaient et amenaient les populations en captivité et les empêchaient de travailler dans leurs champs" (Yaméogo 1990 :13). Selon Mathieu Kaboré, «cette sécurité précaire conduisit les nanamsés de Lay et de Nandiala à 
entreprendre des démarches auprès de Moog-naaba Kuuda afin de pacifier la région » (Kaboré 2014 :7).

À l'origine, le siège du royaume se trouvait à Siglé et s'appelait «Bêgtenga». Mais l'administration coloniale, en commun accord avec le Lallé Naaba de l'époque, a décidé de déplacer le siège dans la ville de Koudougou où résidait l'administrateur colonial (SFG1988 2015 :87). Ce changement a eu lieu sous le règne de Lallé naaba Ligdi (1913-1937) ; dix-huitième chef (Kiendrebeogo 2016 :86). Selon les sources orales et écrites, le Palais actuel serait construit sur les cendres de celui de Naaba Ligdi. Il a été érigé en 1939-1940 par Naaba Tigré, le successeur de Naaba Ligdi.

"Sur le plan architectural, le palais répondait aux critères de construction grécoromaine ou égyptienne. L'essentiel des matériaux de construction étaient le banco et le bois. Les murs sont larges d'environ $60 \mathrm{~cm}$ et construits en adobe avec des briques plus ou moins rectangulaires. Ces murs sont épais et ont la propriété de supporter directement une toiture. Il comporte deux compartiments. C'est un bâtiment à deux niveaux, avec cinq pièces au rez-de-chaussée et deux pièges à l'étage. Actuellement, le palais est en ruine. Malgré son importance historique et culturelle pour le Burkina Faso en général et la commune de Koudougou en particulier, aucune mesure de protection n'est prise. $\mathrm{Vu}$ sa proximité avec la nouvelle route à bitumer, il est nécessaire de prendre des mesures d'atténuation lors des travaux de bitumage. » (SFG1988 2015 :87)

Fig.12. Vue du Palais royal de Lallé

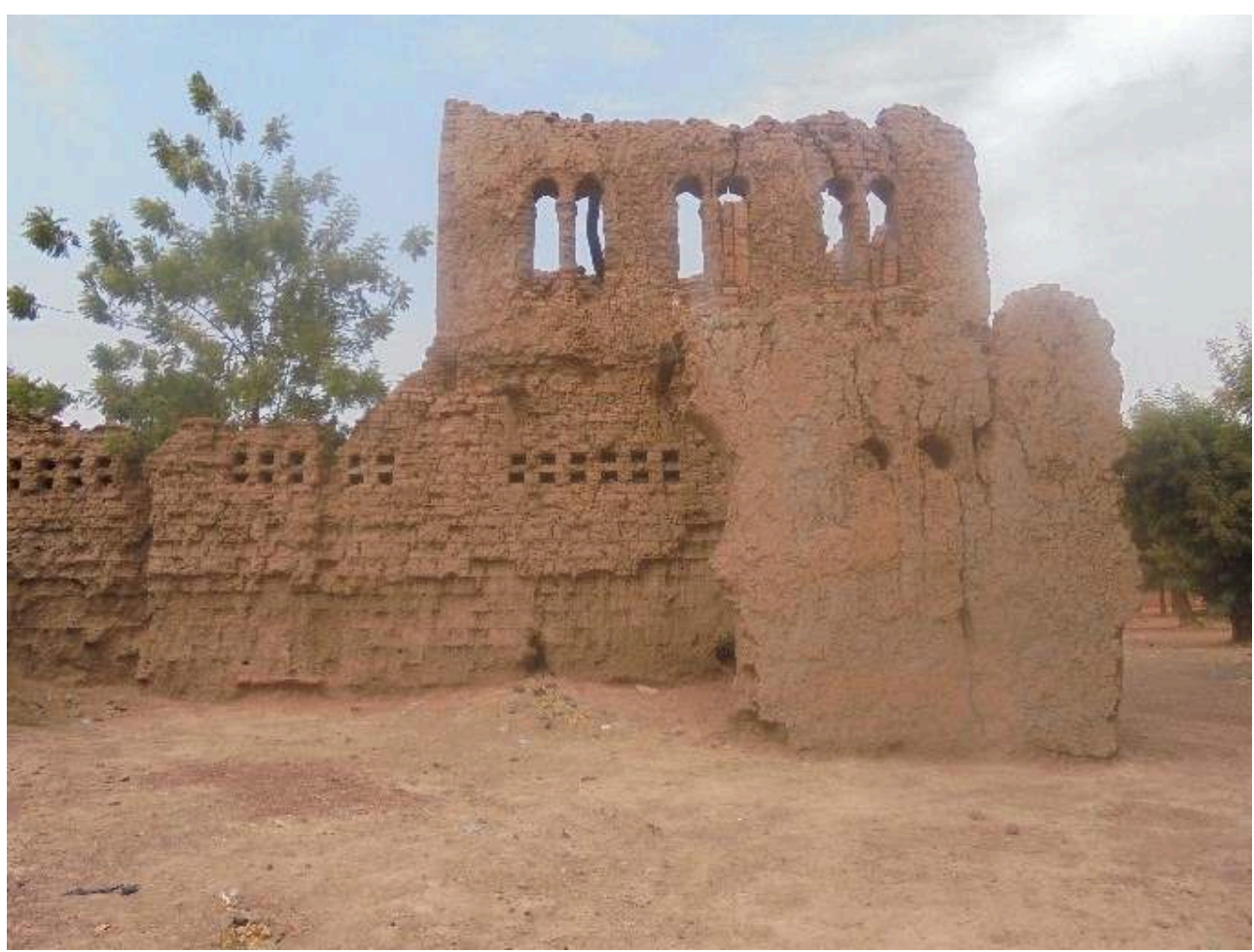

Photos : Birba N., mai 2017

Le deuxième patrimoine historique non moins important de la ville de Koudougou est le Palais de Maurice Yaméogo, premier Président de la Haute Volta, Burkina Faso actuel. En effet, le Palais fut construit en 1964, quatre ans après l'indépendance de la Haute Volta et inauguré en 1965. Le Palais est implanté dans le quartier Dapoya 
(quartier familial de Maurice Yaméogo), au secteur $n^{\circ} 1$ de la ville de Koudougou. Il est érigé sur une aire d'environ quatre hectares.

La cour du palais est assez vaste et comporte deux allées. On y accède par deux portes d'entrée, une à l'Est et l'autre à l'Ouest, à côté desquels se trouvent implantés des portedrapeaux et des postes de contrôle. Le palais se caractérise par une grande bâtisse qui se distingue par son standing et son originalité architecturale. Le bâtiment est un immeuble à deux niveaux construit à base de briques faites en cuirasse et en béton. L'essentiel des matériaux de décoration interne de la bâtisse est composé de marbre. La demeure est constituée d'un vaste salon, de plusieurs chambres, de salles de conférences et d'accueil, d'un réfectoire et de salles de bain. Sur la façade Est de la bâtisse, deux impressionnantes défenses de pachyderme sont solidement fixées.

L'aménagement externe du palais est caractérisé par la présence de deux énormes statues d'éléphant, symboles mythiques du RDA (Rassemblement Démocratique Africain), parti politique du Président Maurice Yaméogo. Ces «éléphants » semblent veiller sur l'entrée du bâtiment principal. À proximité de ces deux formes zoomorphes, une statue à l'effigie du président est facilement reconnaissable.

La construction de ce palais a fait l'objet de plusieurs polémiques quant à l'origine des fonds utilisés. Il demeura la propriété du Président jusqu'au 3 janvier 1966. Son régime est alors renversé par une manifestation populaire, organisée par les forces vives de la nation voltaïque. Suite à cette insurrection populaire, les biens du Président, y compris le palais, sont confisqués par l'État.

Pa la suite, le palais est à nouveau occupé durant la période révolutionnaire et ceci par le Bataillon d'Intervention Aéroportée (BIA) dirigé par le Capitaine Boukary Kaboré dit Le Lion. Ce bataillon y a séjourné pendant deux ans, avant d'être délogé de force, environ deux semaines après la chute tragique du régime du Président Thomas Sankara, le 15 octobre 1987. Mais en 1992, avec le retour à une vie constitutionnelle normale, le palais est restitué à la famille du président, quelques temps avant sa mort, en septembre 1993. Il fut inhumé dans l'enceinte du palais, à l'entrée orientale de la cour.

Dans ces conditions, l'occupation successive du palais depuis sa construction constitue un repère historique des générations actuelles et futures. L'essentiel de l'histoire politique contemporaine y est incrusté.

Depuis lors, le palais est abandonné et se trouve dans un état de dégradation avancé. Il est devenu un dépotoir où les commerçants et les riverains déversent toutes sortes d'ordures. Ainsi, le luxe d'antan a cédé la place à la poussière et aux déchets de toutes sortes. Partout dans la cour, de la tombe du Président à la piscine, ce sont les herbes et les déchets qui dictent leur loi. La toiture de la bâtisse centrale menace de s'effondrer. Dès lors, l'urgence de la réhabilitation du palais n'est plus à démontrer pour servir de support à la création de l'écomusée dans la ville de Koudougou.

Les différents sites patrimoniaux décrits ci-dessus sont dans un état de dégradation avancé dont le principal facteur demeure l'urbanisme que connaît la ville de Koudougou ces dernières années. 
Fig.11. Vue du Palais de Maurice Yaméogo

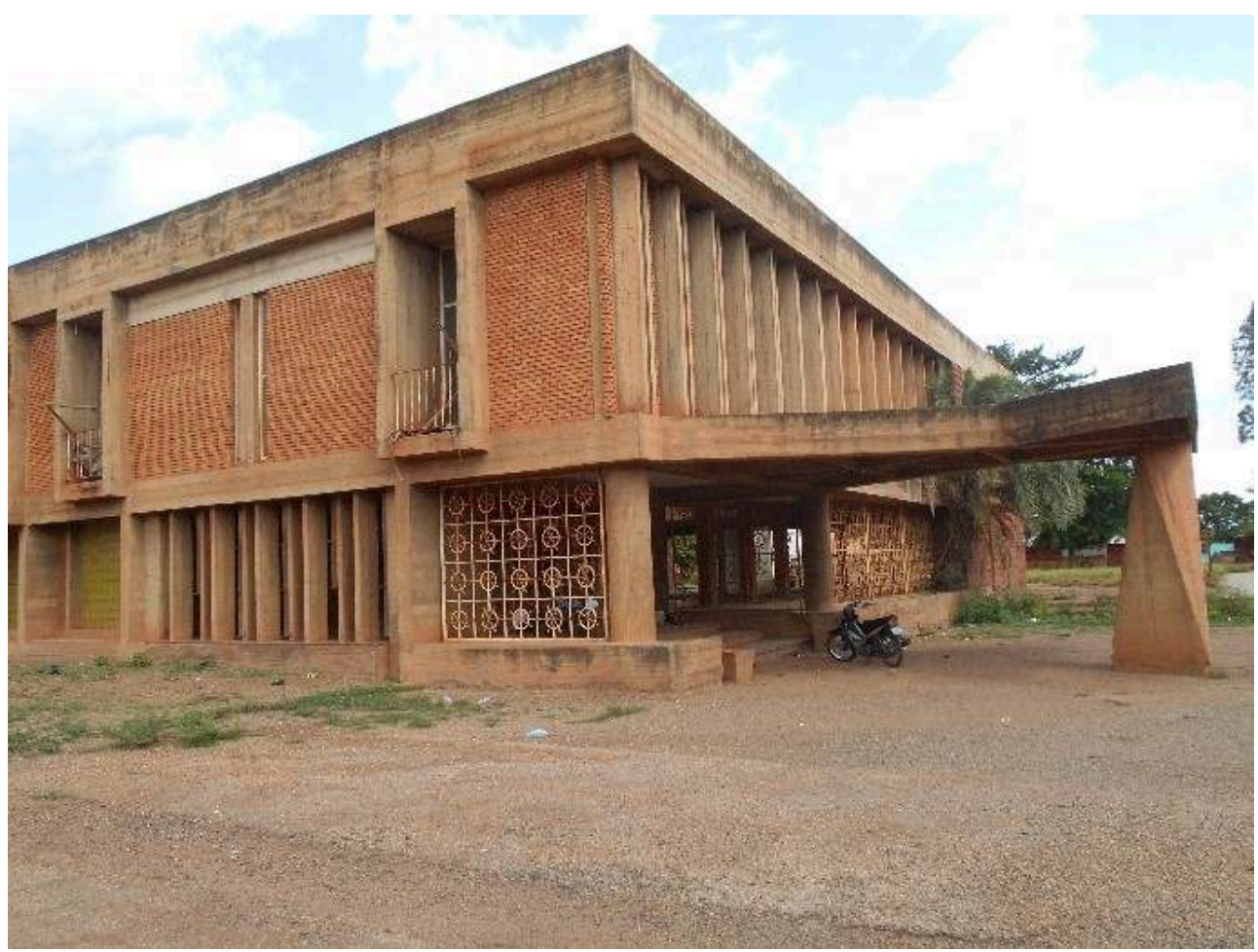

Photos : Birba N., mai 2017

43 L'état de conservation des éléments du patrimoine culturel de la commune de Koudougou n'est pas du tout reluisant. Ces lieux d'expressions et de conservation de la mémoire collective doivent être intégrés dans la reconnaissance des patrimoines nationaux, en relation avec les actions de développement et la promotion du tourisme, pour donner une identité historique et culturelle à la commune. Pour ce faire, cette approche patrimoniale des sites invite à réfléchir sur la mise en œuvre des stratégies adéquates de protection et de valorisation du patrimoine communal. Ainsi, l'inventaire exhaustif des sites de la commune et leur exploitation écomuséale, en impliquant tous les acteurs, semble être la meilleure stratégie de sauvegarde de ce patrimoine.

\section{L'urbanisation, facteur de destruction des sites du patrimoine culturel}

Qu'il s'agisse des paysages culturels (bois sacrés, mares sacrés), les sites archéologiques et les monuments historiques, l'urbanisation galopante de la ville de Koudougou est un facteur de destruction de ces biens culturels. En effet, les sites se retrouvent dans un environnement où les besoins de développement économique, associés aux facteurs climatiques accentuent grandement leur état de dégradation. Dans la perspective des investissements économiques, des infrastructures routières et d'autres grands travaux d'aménagement du territoire sont en projet. La réalisation de ces ouvrages ne sera pas sans conséquence sur les paysages culturels et les sites associés, longtemps et jalousement protégés pour la survie des pratiques culturelles et l'équilibre de l'environnement. 

Le patrimoine est tout ce qui se trouve sur l'axe de l'histoire; il marque les étapes de l'évolution humaine ou se prête, consciemment ou pas, comme la mémoire de celles-ci. Par définition, il est un bien collectif qui raconte l'histoire d'un peuple, d'une ville, d'un territoire et se transmet de génération en génération (Barillet et al. 2006 :26). Dans cette perspective, il est intimement rattaché au passé, donc à l'ancien. Mais dans bien des cas en Afrique, il est victime de cette dernière conception. Il est souvent soit ignoré lors des grands projets d'aménagement du territoire ou simplement détruit. Dans le cas de la ville de Koudougou, l'on remarque que certains sites du patrimoine culturel physique (bois sacrés, sites archéologiques...) ont dû subir les effets néfastes de l'aménagement du territoire. éléments du patrimoine culturel. Des parcelles d'habitation ont été attribuées sur des sites archéologiques, contribuant à leur destruction. De même, dans le cadre du Projet d'Infrastructure, de Développement Urbain et de Mobilité (PIDURMO), financé par la Banque Mondiale, la ville de Koudougou doit bénéficier du bitumage de la voie « 56 ", (liaison RN14-RN21). Longue de $6 \mathrm{~km}$, elle traverse les secteurs 1, 2, 7 et 10. Nos investigations archéologiques et ethnographiques ont permis d'identifier de nombreux bois sacrés et sites historiques qui seront impactés lors des grands travaux de ce bitumage faute de mesures de protection adéquates. Par exemple, les bois sacrés de Goguin de Dapoya, de Zakin et la mare sacrée « rita-samba » ainsi que le lieu de culte du quartier Zinguedeguin seront traversés par la nouvelle voie. Il est urgent que des mesures idoines de protection soient prises en vue de la sauvegarde de ces paysages culturels. Ils ne doivent leur survie qu'au fait d'être associés aux croyances rituelles des communautés locales qui les considèrent comme leurs lieux de culte.

De tout ce qui précède, l'état de conservation des éléments du patrimoine culturel de la commune de Koudougou n'est pas du tout reluisant. Ces lieux d'expression et de conservation de la mémoire collective doivent être intégrés dans la construction des patrimoines nationaux en relation avec les actions de développement et la promotion du tourisme pour donner une identité historique et culturelle de la commune. Pour ce faire, cette approche patrimoniale des sites incite à réfléchir sur la mise en œuvre des stratégies adéquates de protection et de valorisation du patrimoine communal. Ainsi, l'inventaire exhaustif des sites de la commune et leur exploitation écomuséale en impliquant tous les acteurs nous semble être la meilleure stratégie de sauvegarde de ce patrimoine.

\section{De la sauvegarde et la valorisation des sites du patrimoine culturel de la commune de Koudougou}

Le constat d'ensemble sur l'état de conservation des sites du patrimoine culturel et naturel de la commune de Koudougou est qu'ils sont menacés par des facteurs tant naturels qu'anthropiques. Les sites sont influencés fortement par leur environnement immédiat. En effet, la pression démographique accompagnée d'une urbanisation non maîtrisée de la ville de Koudougou constituent les facteurs fondamentaux de dégradation des sites. Lassané Yaméogo fait le constat suivant: «la population de Koudougou a connu une évolution spectaculaire, passant de 15920 habitants en 1936 à 138209 en 2006. Une telle croissance démographique s'est accompagnée d'une 
extension du territoire communal, réduisant ainsi les espaces verts au profit des lotissements et équipements urbains" (Yaméogo 2015). Ainsi, pour faire face à ces différentes menaces du patrimoine culturel, il est nécessaire, d'une part de recenser tous les sites susceptibles de présenter une valeur patrimoniale et historique et, d'autre part, de mener des actions concertées vers une exploitation écomuséale des sites.

\section{De la nécessité de l'inventaire des sites du patrimoine culturel}

Pour protéger et valoriser un patrimoine, qu'il soit culturel ou naturel, il faut d'abord l'identifier. Pour ce faire, l'inventaire est la démarche qui consiste à recenser sur une aire donnée, géographique ou culturelle, les éléments du patrimoine culturel: monuments historiques, sites archéologiques, mausolées, festivals, sanctuaires, paysages culturels. Ces éléments peuvent appartenir à l'État, à une collectivité territoriale et même à une personne physique, etc.

Cela signifie que la première condition sine qua non à tout projet de protection et de valorisation du patrimoine culturel réside dans la connaissance complète des sites à sauvegarder. L'inventaire demeure un instrument de protection du patrimoine dans toutes ses facettes. Nos recherches nous ont permis d'identifier un nombre important de sites dans la ville de Koudougou, mais il faut remarquer qu'il reste beaucoup à faire. Sur presque toute l'étendue du territoire communal, de nombreux sites patrimoniaux restent encore à découvrir. Aussi, pour créer un projet de valorisation de ce patrimoine, il est nécessaire de l'intégrer dans un vaste programme d'inventaire pouvant prendre en compte les autres potentialités culturelles des villages rattachés à la commune.

51 Pour le cas spécifique des bois sacrés, la commune doit mettre en place une politique efficace permettant de faire une rigoureuse identification de ses espaces culturels et naturels. Dans certains pays d'Afrique comme le Bénin, le Ghana, le Kenya, des actions d'inventaire des bois sacrés ont déjà été entreprises. "Cependant, un inventaire des bois sacrés doit tenir compte du concept de « paysage culturel » (Elamé 2003) et doit se baser sur une approche participative, c'est-à-dire l'implication forte des populations autochtones, gardiens et propriétaires de ces espaces magico-religieux.

Pour les sites associés, notamment les sites archéologiques et historiques, des prospections extensives et intensives doivent être menées à l'échelle communale afin de pouvoir dresser une carte des biens culturels et de déterminer les sites significatifs, tant du point de vue de l'état de conservation que du point de vue scientifique et patrimonial. Cela permettra d'identifier les sites à protéger contre certains dangers tels que les grands travaux d'aménagements et les pratiques culturales peu soucieuses de ce patrimoine. Aussi, seul un inventaire exhaustif et rigoureux pourra permettre de connaître et de proposer l'inscription des sites les plus importants sur la liste du patrimoine communal et peut-être sur la liste indicative nationale.

Après l'inventaire exhaustif des sites, il est également nécessaire de procéder à des actions concrètes de leur valorisation telle que l'exploitation écomuséale. Cette démarche pourrait contribuer à une prise de conscience de la part des acteurs pour une prise en compte du patrimoine culturel et naturel dans les politiques de développement durable et d'aménagement du territoire. 


\section{Vers une exploitation écomuséale des sites patrimoniaux de la commune de Koudougou} institution culturelle encore méconnue dans notre pays. Ainsi, l'écomusée peut être considéré comme un espace non homogène, culturellement parlant, «couvrant la totalité d'un territoire plus ou moins cohérent, utilisant le patrimoine global de ce territoire et l'expertise de ses habitants. C'est un instrument culturel pour le développement culturel du territoire, selon un processus continu impliquant la communauté locale, les collectivités territoriales et les institutions administratives et scientifiques et un certain nombre de professionnels. L'écomusée peut être qualifié de musée communautaire, lorsque réellement la communauté est associée à toutes les phases du processus, de musée de territoire, lorsque le musée traite de tout un territoire sous ses différents aspects, pour servir à son développement » (Varine 2006 : 10). La mise en œuvre de l'écomusée recommande donc l'implication effective de tous les acteurs.

56 Ainsi, les autorités coutumières, gardiennes des bois sacrés et des sites associés, les autorités décentralisées ainsi que les services déconcentrés de l'État, notamment la Direction régionale de la culture et du Tourisme du Centre Ouest doivent mutualiser leurs actions dans le cadre de l'exploitation écomuséale des sites. Cette gestion concertée et participative est importante pour la constitution d'un écomusée dont la première vocation serait la promotion du patrimoine culturel et touristique, levier du local et durable. En outre, les communautés locales doivent être les premières dans une politique de création d'écomusée car l'appropriation de la notion de patrimoine par les habitants d'une ville ou d'une contrée et la prise de conscience de sa valeur représentent un enjeu majeur pour la société. Pour ce faire, une politique de sensibilisation est nécessaire et doit être instaurée, par exemple grâce à des manifestations telles que les Journées du Patrimoine Communal, à l'occasion desquelles on observe un intérêt grandissant de la part des habitants pour découvrir ou redécouvrir leur patrimoine.

Dans le cas spécifique des sites de la commune de Koudougou, en plus de l'implication des acteurs, il faut établir une bonne stratégie managériale avec des experts compétents afin qu'ils proposent des méthodes d'aménagement écomuséal qui tiennent compte des spécificités des sites identifiés. Cette expertise devra être réalisée par une équipe de recherche interdisciplinaire aguerrie aux questions d'aménagement du 
territoire et du patrimoine. Le succès du projet dépend de l'implication effective des communautés locales.

\section{Conclusion}

La commune de Koudougou possède des potentialités culturelles de grande importance. Constitué de paysages culturels (bois sacrés), des sites archéologiques et des monuments historiques, ce patrimoine est un héritage ancestral qui se doit d'être conservé au profit des générations futures. En dépit des menaces auxquelles il est confronté, le patrimoine culturel de Koudougou reste prometteur dans le paysage touristique et culturel du Burkina Faso.

Des actions concrètes de protection doivent en outre être entreprises afin de sauvegarder ce riche patrimoine. En premier lieu, l'inventaire exhaustif des sites dans l'espace communal s'avère un impératif. Cet inventaire permettra de disposer d'une liste complète des sites du patrimoine communal. La deuxième action d'importance de sauvegarde des sites est leur mise en valeur à des fins touristiques, à travers la création d'un écomusée, en intégrant tous les aspects culturels de la commune.

Toutes ces actions doivent être menées en impliquant toutes les parties prenantes, notamment les communautés locales. L'implication et la responsabilisation des populations locales dans l'étude des différents sites permettent d'envisager leur participation au-delà de la pure information et leur attribuer un rôle prépondérant dans la protection et la mise en valeur des sites. C'est dans ce sens que l'ICOMOS, dans sa déclaration de Québec sur la sauvegarde de l'esprit du lieu, stipule que: " reconnaissant que l'esprit du lieu est transmis essentiellement par des personnes et que la transmission participe activement à sa conservation, nous déclarons que c'est par la communication interactive et la participation des communautés concernées que l'esprit du lieu est sauvegardé, employé, enrichi. La communication permet ainsi de garder l'esprit du lieu vivant. (...). Considérant que les communautés locales sont généralement les mieux placées pour saisir l'esprit du lieu, surtout dans le cas des groupes culturels traditionnels, nous soutenons qu'elles devraient être intimement associées à tous les efforts de conservation et de transmission de l'esprit du lieu ${ }^{8}$ ». Pour lutter efficacement en faveur de la protection des sites patrimoniaux, il faut définir des méthodes d'action tendant à impliquer et à responsabiliser les populations locales. Toute action de sauvegarde du patrimoine, sans prise de conscience de la population et sans sa participation active, est une entreprise illusoire.

\section{BIBLIOGRAPHIE}

ADJANOHOUN Édouard, «La notion de paysage culturel et les liens nature-culture en Afrique », in C. LE COUR GRAND-MAISON et G. SAOUMA-FORERO (dir.), Le patrimoine culturel africain et la convention du patrimoine mondial, Porto-Novo, Quatrième réunion de stratégie globale, Unesco, 1998, p.69-84 
BARILLET Christian, JOFFROY Thierry, LONGUET Isabelle (dirs.) Patrimoine culturel et développement local. Guide à l'attention des collectivités locales africaines, Paris, Édition CRATerreENSAG/Convention France-UNESCO, 2006

BATIÉNO D., Le patrimoine culturel physique du sheku (territoire) de Didyr et environs, des origines à 1912. Mémoire de Maitrise d'Histoire et Archéologie, Département d'Histoire et Archéologie, Université de Koudougou, 2013

BIRBA Noaga, « La paléométallurgie du fer dans la province du Bam (Burkina Faso) : identité des acteurs et mobilité des techniques ", in C. ROBION-BRUN\& B. MARTINELLI (éds.), Métallurgie du fer et Sociétés africaines. Bilans et nouveaux paradigmes dans la recherche anthropologique et archéologique, Cambridge, Monographs in African Archaeology, 81, séries 2395, 2012, p.177-184

BIRBA Noaga, « Le patrimoine archéologique dans le Parc national des Deux-Balé (Burkina-Faso », Revue Africaine d'Anthropologie, Nyansa Pô, n¹6, 2014, p.78-101

ELAMÉ Esoh, « Plaider pour une protection des bois sacrés en Afrique noire », Mémoire soumis au XII Congrès forestier mondial, Québec City, Canada, 2003, en ligne : http://www.fao.org/3/XII/ 1006-A3.htm\#P10_167

HILGERS Mathieu, « Les conflits autour de l'histoire de Koudougou (Burkina Faso) », Cahiers d'études africaines, $\mathrm{n}^{\circ} 186,2007, \mathrm{p} .313-344$

IZARD Michel, Introduction à l'histoire des royaumes mossis, Paris, Éditions du CNRS-CVRS, 1970

JUHÉ-BEAULATON Dominique, « Des bois sacrés vodou aux paysages culturels : la conservation de la bio-diversité en question (Sud Togo et Bénin) », Rapport final IFB 'bois sacrés et conservation de la biodiversité', 2006, halshs-00343524v2

JUHÉ-BEAULATON Dominique, Forêt sacrées et sanctuaires boisés : des créations culturelles et biologiques (Burkina Faso, Togo, Bénin), Paris, Karthala, 2010

KABORÉ M., Rôle de la chefferie traditionnelle de Lallé dans l'implantation de l'Eglise Catholique à Koudougou et son impact social (1897-1970), Mémoire de maîtrise, Département d'Histoire et Archéologie, Université de Koudougou, 2014

KIENDRÉBÉOGO F., Prospections archéologiques dans l'ancien Kombéré de Lallé (Burkina Faso), Mémoire de Master 2, Département d'Histoire et Archéologie, Université Ouaga I, Professeur Joseph KiZerbo, 2018

KIÉNON-KABORÉ Timpoko Hélène, La métallurgie ancienne du fer au Burkina Faso : province de Bulkiemdé. Approche ethnologique, historique, archéologique et métallographique. Un apport à l'histoire des techniques en Afrique, Paris, Éditions de l'Harmattan, 2003

KOHLER Jean-Marie, « Activités agricoles et transformations socio-économiques dans une région de l'ouest Mossi », Bulletin de Liaison Sciences Humaines-ORSTOM, 1966, n 4, p.87-93

KOTÉ Lassina, « Paléométallurgie du fer dans la boucle du Mouhoun », in DOREL-FERRÉ Gracia (dir.), Les arts du feu en Champagne-Ardenne et ailleurs, APIC, 2004, p.11-22

KOTÉ Lassina, « Archéologie, patrimoine et promotion touristique dans la région du centre nord au Burkina Faso ", Annales de l'Université de Ouagadougou, Série Lettres et sciences Humaines, 2012, p. 268-306

KOTÉ Lassina, « Archéologie préventive et exploitation minière au Burkina Faso : le cas de Perkoa », in Journal africain des sciences de l'environnement (JASE), 2012, p.45-61 
LE BERRE Michel, « La genèse des paysages culturels africains », in La convention du patrimoine et les paysages culturels en Afrique, réunion d'experts-Tiwi. Kenya : 9-14 mars 1999, Unesco, CRAterre, 2000, p.44-58

RIVIERE Georges-Henri, « Les écomusées : retour à la définition et évolution », Paris, Revue d'art, 1989

SANOU Serge Stéphane, Archéologie et histoire du peuplement de la province du Boulkiiémdé (région du centre-ouest du Burkina Faso), Mémoire de Master 2, Département d'Histoire et Archéologie, Université Ouagadougou I, Professeur Joseph Ki-Zebo, 2017

SFG 1988, Cadre de gestion des ressources culturelles physiques (CGRCP), Ouagadougou, Ministère des Infrastructures, du Désenclavement et des Transports, 2015

TIENDRÉBÉOGO Yamba, « Histoire traditionnelle des Mossi de Ouagadougou », Journal de la Société des Africanistes, 1963, tome 33, fascicule 1, 1963, p.7-46

VARINE Hugues de, Écomusée : un mot, deux concepts, mille pratiques, 2006, www.huguesdevarine.eu/img/cms/2006\%20

YAMÉOGO É., La politique française de développement des cultures d'exportations dans lecercle de Koudougou (1924-1947), Mémoire de maîtrise, Université de Ouagadougou, 1990

YAMÉOGO Lassané, « Le patrimoine méconnu des bois sacrés de la ville de Koudougou (Burkina Faso) : de la reconnaissance à la sauvegarde ", Cahiers de géographie du Québec, vol.59, n¹66, 2015, p.71-90

\section{ANNEXES}

Les sources orales

\begin{tabular}{|c|c|c|c|c|c|}
\hline $\begin{array}{l}\text { Nom- } \\
\text { Prénom (s) }\end{array}$ & Age & Profession & Statut social & $\begin{array}{l}\text { Lieu de } \\
\text { l'enquête }\end{array}$ & $\begin{array}{ll}\text { Date de } \\
\text { l'enquête }\end{array}$ \\
\hline Naaba Warga & $\begin{array}{l}68 \\
\text { ans }\end{array}$ & Cultivateur & $\begin{array}{l}\text { Chef de terre du quartier } \\
\text { Sougpelcé }\end{array}$ & & $24 / 07 / 2017$ \\
\hline $\begin{array}{l}\text { Yaméogo } \\
\text { Benjamin }\end{array}$ & $\begin{array}{l}53 \\
\text { ans }\end{array}$ & Cultivateur & - & & 09/07/2017 \\
\hline $\begin{array}{l}\text { Yaméogo } \\
\text { Tenga }\end{array}$ & 75 & Cultivateur & $\begin{array}{l}\text { Responsable coutumier du } \\
\text { quartier Bourkina }\end{array}$ & & $16 / 04 / 2017$ \\
\hline $\begin{array}{l}\text { Yaméogo } \\
\text { Z.Michel }\end{array}$ & $\begin{array}{l}79 \\
\text { ans }\end{array}$ & Cultivateur & $\begin{array}{l}\text { Responsable coutumier du } \\
\text { quartier Zinguedeguin }\end{array}$ & & 07/07/2017 \\
\hline Zongo Joseph & $\begin{array}{l}62 \\
\text { ans }\end{array}$ & Cultivateur & - & & $15 / 08 / 2016$ \\
\hline Zongo Paul & $\begin{array}{l}63 \\
\text { ans }\end{array}$ & Cultivateur & - & & $15 / 08 / 20$ \\
\hline
\end{tabular}




\begin{tabular}{|l|l|l|l|l|l|}
\hline Zongo Tenga & $\begin{array}{l}70 \\
\text { ans }\end{array}$ & $\begin{array}{l}\text { Cultivateur- } \\
\text { forgeron }\end{array}$ & Responsable de forge. & 17/07/2017 \\
\hline Zongo Tiga & $\begin{array}{l}69 \\
\text { ans }\end{array}$ & Cultivateur & $\begin{array}{l}\text { Responsable coutumier du } \\
\text { quartier Sougpecé }\end{array}$ & $02 / 08 / 2016$ \\
\hline
\end{tabular}

\section{NOTES}

1. Les Engagements Nationaux constituent un vaste programme lancé le 02 juin 1994 par le président Blaise Compaoré dont la mise en œuvre est sensée contribuer à réduire fortement la pauvreté par l'intensification des productions. Cette politique s'articule autour de six principaux engagements dont le premier est «la sauvegarde de l'environnement et la lutte contre la désertification $"$.

2. Des sacrifices propitiatoires y sont souvent effectués. La notion d'économie doit être perçue ici sous l'angle traditionnel du terme. Les cultes assurent aux populations une bonne pluviométrie et de bonnes récoltes.

3. Tenga Yaméogo : entretien du 16/04/2017 à Koudougou.

4. Tiga Zongo : entretien du 02/08/2016 à Koudougou.

5. Convention-cadre du Conseil de l'Europe relative à la valeur du patrimoine culturel pour la société, dite Convention de Faro, en date du 27 octobre 2005.

6. Naaba Kumdumyé (1540 ?-1562 ? 1563 ?), chronologie de Michel Izard.

7. Naaba Kuuda (1562 ?1563 ?-1584 ?1585 ?), chronologie de Michel Izard.

8. ICOMOS, Déclaration de Québec, sur la sauvegarde de l'esprit du lieu, Québec, 4 octobre 2008.

\section{RÉSUMÉS}

À l'instar des autres collectivités territoriales burkinabé, la commune de Koudougou regorge de riches patrimoines naturels, archéologiques et historiques. Chaque secteur et village de la commune possède ses sites patrimoniaux. Ces biens culturels constituent des repères historiques des communautés locales. Chargés d'histoire, ils révèlent aussi bien les grandes étapes de la formation des peuples que leur évolution dans le temps. Ils passent pour être des âtres de la promotion touristique pour peu qu'ils soient pris en compte dans les politiques nationales de développement. Ils sont cependant, dans leur grande majorité, menacés de destruction face aux travaux d'aménagement intensifs et d'autres facteurs anthropiques. Dans cette étude, nous verrons comment s'opère le processus de protection et de valorisation malgré la multitude de défis qui menacent ce patrimoine. Nous présenterons d'abord les bois sacrés et les sites associés, puis nous décrirons les mesures de protection et de valorisation envisagées dans un contexte marqué par l'abandon des cultures locales au profit d'une «modernité » très peu soucieuse de respecter les valeurs culturelles ancestrales.

Like other territorial collectivities of Burkina Faso, the municipality of Koudougou abounds in rich natural, archaeological and historical heritages. Each sector and village have its heritage sites. These cultural goods constitute important historical references to local communities. Filled 
with history, these cultural goods reveal the main steps of the formation of local populations and their evolution. They could also contribute to local tourism promotion if taken into account in national development policies. However, most of these heritages are threatened of destruction with the progress of intensive planning works and other anthropogenic factors. In this study, we analyse the issue of the protection and the valorization of sacred forests and their associated sites, in a context of local cultures neglected at the advantage of the so-called "modernity", that is, for the most, irrespective of ancestral cultural values.

\section{INDEX}

Mots-clés : histoire des techniques, écomusée, patrimoine, paysage culturel

Keywords : history of technology, eco-museum, heritage, cultural landscape, cultural sustainability

\section{AUTEUR}

\section{NOAGA BIRBA}

Noaga Birba est enseignant-chercheur au Département d'Histoire et Archéologie à l'Université Norbert Zongo (Burkina Faso). Ses axes de recherche : patrimoine culturel et développement durable, la sidérurgie ancienne et l'archéologie préventive. Il a réalisé plusieurs études dans ces différents domaines et publiés des articles scientifiques. 